\title{
Study of a 3D diamond detector with photon and proton micro-beams
}

DOI:

10.1016/j.diamond.2016.02.005

\section{Document Version}

Accepted author manuscript

Link to publication record in Manchester Research Explorer

\section{Citation for published version (APA):}

Forcolin, G., Grilj, V., Hamilton, B., Li, L., Mcgowan, M., Murphy, S. A., Oh, A., Skukan, N., Whitehead, D., \& Zadoroshnyj, A. (2016). Study of a 3D diamond detector with photon and proton micro-beams. Diamond and Related Materials, 65, 75-82. https://doi.org/10.1016/j.diamond.2016.02.005

\section{Published in:}

Diamond and Related Materials

\section{Citing this paper}

Please note that where the full-text provided on Manchester Research Explorer is the Author Accepted Manuscript or Proof version this may differ from the final Published version. If citing, it is advised that you check and use the publisher's definitive version.

\section{General rights}

Copyright and moral rights for the publications made accessible in the Research Explorer are retained by the authors and/or other copyright owners and it is a condition of accessing publications that users recognise and abide by the legal requirements associated with these rights.

\section{Takedown policy}

If you believe that this document breaches copyright please refer to the University of Manchester's Takedown Procedures [http://man.ac.uk/04Y6Bo] or contact uml.scholarlycommunications@manchester.ac.uk providing relevant details, so we can investigate your claim.

\section{OPEN ACCESS}


Study of a 3D diamond detector with photon and proton micro-beams • January $2016 \bullet$ Diamond and Related Materials

\title{
Study of a 3D diamond detector with photon and proton micro-beams
}

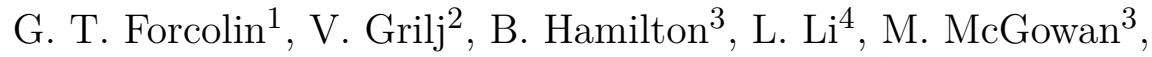 \\ S. A. Murphy ${ }^{1 *}$ A. Oh ${ }^{1}$, N. Skukan ${ }^{2}$, D. Whitehead ${ }^{4}$, A. Zadoroshnyj ${ }^{5}$ \\ 1. School of Physics and Astronomy, University of Manchester, UK \\ 2. Ruđer Bošković Institute, Zagreb, Croatia \\ 3. School of Electrical and Electronic Engineering, University of Manchester, UK \\ 4. School of Mechanical, Aerospace, and Civil Engineering, University of Manchester, UK \\ 5. School of Materials, University of Manchester, UK
}

\begin{abstract}
To form a 3D diamond detector electrodes were produced in diamond by a femtosecond laserinduced phase transition of diamond to graphite. The process parameters were varied to study the influence on electrode resistivity and induced stress. A technique for a relative measurement of stress induced in 3D diamond detectors is described. The detector was characterised with a $15 \mathrm{keV}$ photon micro-beam (Diamond Light Source, Oxford) and a $4 \mathrm{MeV}$ proton micro-beam (Ruđer Bošković Institute, Zagreb). The detector shows characteristics consistent with full charge collection. Spatially resolved transient current measurements were obtained with protons for the first time, and the results were compared to simulations of the detector.
\end{abstract}

\section{INTRODUCTION}

Since many years synthetic diamond is used for particle detection applications in collider experiments, in synchroton radiation sources and for dosimetric applications in medical radiation therapy. Recently a new type of diamond particle detector with in-bulk electrodes has been demonstrated to be feasible [1]. The so called $3 \mathrm{D}$ detector geometry promises to have superior radiation hardness compared to the conventional planar electrode geometry. 3D prototype detectors have been investigated with beta particles [2], high energy protons [3], alpha particles [4], and irradiation studies with neutrons seem to confirm the improved radiation hardness [5].

In this paper the process parameters to produce a $3 \mathrm{D}$ diamond detectors are varied, and the structural, electrical and detector properties are investigated. Section 2 describes the production process of the 3D diamond test detector and structural characterisation results. In Section 3 the results of an investigation with a synchrotron beam are presented. Section 4 presents studies of the charge collection properties with a proton micro beam including spatially resolved transient currents. Finally, experimental data are compared to simulation results in Section 5 .

\section{Detector FABrication}

The electrodes in diamond are fabricated by inducing a phase transition of the diamond lattice using a short $(<\mathrm{ns})$ pulse length laser. The electrodes formed through this technique consist of a combination of graphite and amorphous carbon [6]. It has been shown that picosecond and femtosecond lasers are capable of inducing this phase transition, with femtosecond lasers producing well-defined electrodes [7].

The electrodes were produced with this laser process [6] using a Coherent Libra Ti-Sapphire femtosecond laser, with a $100 \mathrm{fs}$ pulse duration

*Corresponding author: steven.murphy@manchester.ac.uk 
Study of a 3D diamond detector with photon and proton micro-beams • January $2016 \bullet$ Diamond and Related Materials

at a wavelength of $800 \mathrm{~nm}$, operating in $\mathrm{TEM}_{00}$ beam mode and a repetition rate of $1 \mathrm{kHz}[8$. The diamond sample was mounted to a programmable 3 -axis stage to control the location of the focal point of the beam within the diamond sample. The translational speed was set to $20 \mu \mathrm{m} / \mathrm{s}$, with energy densities varying from $2-4.8 \mathrm{~J} / \mathrm{cm}^{2}$. The laser power was adjusted using a neutral-density filter attenuator, and the laser beam was focused to a diameter of $6 \mu \mathrm{m}$. Four arrays of electrodes with different energy densities were produced. The laser processing was performed from the back of the sample to the front to avoid absorption of light by the electrode itself.

A structured metallisation pattern was applied to the detector by using photolithography, and a sputtering and lift-off process. The sample was annealed at $250{ }^{\circ} \mathrm{C}$ for 5 minutes in a nitrogen atmosphere. A strip pattern connecting the graphitic electrodes was deposited on one surface of the diamond. On the opposite side small pads of metal were deposited to measure the electrical properties of the electrodes. The metallisation contacts consist of a layer of $35 \mathrm{~nm}$ thick titanium, which provided good adhesion to the diamond, and a layer of $60-70 \mathrm{~nm}$ thick gold, which prevents oxidisation of the titanium layer.

With the fabrication technique described a 3D diamond detector was produced from single crystal electronic-grade sample obtained from Element Six [9], with dimensions $4.7 \times 4.7 \times$ $0.5 \mathrm{~mm}^{3}$. The sample was mounted on a printed circuit board with bond wires made of $25 \mu \mathrm{m}$ thick gold. Four arrays of 3D detector cells with a outer dimension of $600 \times 600 \mathrm{\mu m}^{2}$ (Figure $1 \mathrm{a}$, and a cell size of $120 \times 120 \mathrm{\mu m}^{2}$ (Figure 1b) have been produced. Each cell consists of four bias electrodes, with a central readout electrode. Readout electrodes were connected together to form strips and are read out with a single line. The graphitic electrodes were formed with an average diameters varying between $8-15 \mu \mathrm{m}$ depending on the processing parameter and are shown in Figure 1c. For a total of 32 electrodes the formation of a secondary electrode was observed (Figure $1 \mathrm{c}$ right) due to an astigmatism in the beam.

The structure of the electrode at the surface was imaged using a Scanning Electron Microscope (SEM). Figure 1d shows that the electrode fabrication process causes excess material to form on surface of the sample where laser processing begins (seed side). Craters are observed on the surface of the sample where laser processing finishes (exit side). The structural effects seen on the seed and exit side of the diamond are due to the lower density of the electrode material compared to the diamond. The electrode formation causes pressure inside the diamond sample, and near the exit side the pressure build-up is sufficiently high to eject a few microns of diamond material at the surface forming a crater.

Current voltage (I-V) curves were measured for the electrodes in each array using a Keithley 2410 SourceMeter [10. The average electrode diameter and the average resistivity are shown in Table 1. The electrodes exhibit an ohmic behaviour until approximately $\pm 200 \mathrm{~V}$, and show an exponential increase for higher voltages.

In some cases electrodes showed a high resistance and exponential current increase at low voltages indicating a discontinuity in the electrode formation. In array A only all but one electrode showed this behaviour, while in arrays $\mathrm{B}, \mathrm{C}$, and $\mathrm{D}, 6,1$, and 0 electrodes, respectively, were identified as non-ohmic. The average resistance of these non-ohmic electrodes is $200 \mathrm{G} \Omega$ (compared to $100 \mathrm{k} \Omega$ for the ohmic electrodes), and the average resistance between two unconnected electrodes (the diamond bulk) across all four arrays is $158-216 \mathrm{G} \Omega$. The measurements indicate that most of the electrodes do not completely penetrate the diamond bulk in array A.

The material composition of the electrodes was determined using a HORIBA LabRAM HR Evolution Raman Spectrometer [11, with a HeNe laser at a wavelength of $633 \mathrm{~nm}$ and spot size of $5 \mu \mathrm{m}$. Figure 2 shows two clear peaks in the Raman shift range shown (at $1320 \mathrm{~cm}^{-1}$ and $1580 \mathrm{~cm}^{-1}$ ). Crystalline graphite would have a single peak in the Raman shift range shown (corresponding to the $\mathrm{G}$ band at $1583 \mathrm{~cm}^{-1}$ ), and amorphous graphite has two peaks (the D band at $1350 \mathrm{~cm}^{-1}$ and the $\mathrm{G}$ band peak) [12]. The structure formed from the electrode fabrication technique is disorganised graphite, with peaks at $1321 \pm 50 \mathrm{~cm}^{-1}$ and $1578 \pm 30 \mathrm{~cm}^{-1}$. The probe 
Study of a 3D diamond detector with photon and proton micro-beams • January $2016 \bullet$ Diamond and Related Materials

beam is smaller than the electrode diameter, and we do not observe a diamond peak on the electrode area (recognisable by a sharp, narrow peak at $\left.1330 \mathrm{~cm}^{-1}\right)$.

Table 1: Average electrode diameter and resistivity for each array.

\begin{tabular}{|c|c|c|c|}
\hline Array & $\begin{array}{l}\text { Fluence } \\
\mathrm{Jcm}^{-2}\end{array}$ & $\begin{array}{l}\text { Diameter } \\
\qquad \mu \mathrm{m}\end{array}$ & $\begin{array}{c}\text { Resistivity } \\
\Omega \mathrm{cm}\end{array}$ \\
\hline A & 2.0 & $7.6 \pm 0.8$ & 0.75 \\
\hline B & 3.0 & $10.2 \pm 1.2$ & $2.47 \pm 0.86$ \\
\hline $\mathrm{C}$ & 3.5 & $12.4 \pm 0.9$ & $2.58 \pm 0.89$ \\
\hline $\mathrm{D}$ & 4.8 & $18.0 \pm 1.3$ & $2.63 \pm 0.71$ \\
\hline
\end{tabular}

Partially formed electrodes are expected to have less surrounding stress than fully formed electrodes, which can be imaged with a crosspolarised microscope (see Figure 3a). The crosspolarised image shows two partially formed electrodes (red and green), confirmed by optical inspection in Figures $3 \mathrm{~b}$ and $3 \mathrm{c}$ Figures $3 \mathrm{a}$ and $3 \mathrm{c}$ show the stress increases with penetration distance through the diamond bulk, with some stress induced by the partially formed electrode and almost no stress induced due to the electrode in Figure 3b. The number of electrodes that fully penetrate the diamond bulk along with the number of ohmic electrodes is used to determine the percentage yield for electrode formation in Table 1 .

Cross-polarised images for each array are used to calculate the relative stress of the arrays. The brightness of the image is proportional to the stress in diamond and can be used as relative measure of stress. A brightness histogram was obtained for each array, and pixels above a brightness threshold of 200 (with a maximum brightness of 255) were used in the relative stress calculations. These stress measurements are relative to array A. Figure 4 shows the electrode diameter and relative stress both linearly increase with the fluence used to process the graphitic electrodes.

\section{Photon test BeAm DATA}

The beam-line B16 [13] at the Diamond Light Source in Oxfordshire was used to study the detector by X-ray beam induced current (XBIC). The beam-line produced a monochromatic beam of photons with an energy of $15 \mathrm{keV}$, and had a full width half maximum (FWHM) of $5 \mu \mathrm{m}$. The photon absorption length in diamond is approximately $4 \mathrm{~mm}$, therefore there is negligible attenuation of the beam. The photon beam flux was determined using an ionisation Yieldchamber and was attenuated to the order of $10^{6}$ $\%$ photons $s^{-1}$ using aluminium absorbers.

3 The detector was mounted in a metal box 83 with an aluminium foil entrance window to 97 shield against light and electromagnetic noise [1]. 100 The photon beam was incident perpendicular to the detector surface. Data were taken at bias voltages of $\pm 5 \mathrm{~V}, \pm 40 \mathrm{~V}$, and $\pm 60 \mathrm{~V}$. Only arrays $\mathrm{A}$ and $\mathrm{C}$ on the detector were tested due to limitations in the beam time, with two adjacent readout strips per array.

The detector response was studied by measuring the beam induced current. Two current amplifier were used (Keithley 428) to measure the signals of two read-out channels, a DAQ system was recording the current measurements of the device under test, the beam position, the current of the ionization chamber, and the ring current of the accelerator. The expected induced charge in the sample was estimated by calculating the number of absorbed photons per unit time, the energy to create an electron-hole pair is set to $13 \mathrm{eV}$ [14. The gain of the current amplifiers were set to $10^{9}$.

The signal response as a function of position of two adjacent readout lines for array A are shown in Figure 5 Figures $5 \mathrm{a}$ and $5 \mathrm{~b}$ show the same readout line at different bias voltages. The average signal for each of the cells is consistent to within $10 \%$. A more uniform response is observed at higher bias voltages. The signal collected by the electrode on the surface is up to $30 \%$ at $\pm 5 \mathrm{~V}$. At higher bias voltages, the fraction of signal picked up is higher $(50 \%)$ due to the increased electrical field strength in the diamond and the long lifetime of charge carriers in the diamond sample. A crossing bond-wire is visible in Figures $5 \mathrm{c}$ and $5 \mathrm{~d}$. The attenuation due to the gold wire is estimated to be about a factor 2500 [15] hence the "shadow" of the wire is visible. 
Study of a 3D diamond detector with photon and proton micro-beams • January $2016 \bullet$ Diamond and Related Materials

\section{PROTON TEST BEAM}

The proton microbeam [16] at the Ruđer Bošković Institute in Zagreb was used to study the detector by ion beam induced charge (IBIC). The beam produced protons with an energy of $4 \mathrm{MeV}$ in a narrow beam with an FWHM of $1 \mu \mathrm{m}$. The proton range in diamond is approximately $78 \mu \mathrm{m}$ at $4 \mathrm{MeV}$ [17, so protons will stop within the diamond bulk. Data were taken at bias voltages of $\pm 5 \mathrm{~V}, \pm 40 \mathrm{~V}$, and $\pm 60 \mathrm{~V}$. Only array A was tested on the detector. IBIC and timeresolved ion beam induced current (TRIBIC) data were obtained using a self-trigger on the signal. The same detector area on array A tested at Diamond Light Source were investigated with IBIC. The charge was normalised by comparing to a silicon detector.

\subsection{IBIC Results}

The response to the proton micro beam on two adjacent readout lines for array A are shown in Figure 6 Figures 6a and 6b show readout one at different bias voltages. Figures $6 \mathrm{c}$ and 6d show the adjacent readout strip where the shadow of the bond wire is clearly visible. The variation in the charge collected across one cell is shown in Figure 7 .

Comparing the photon and proton data in Figures 5a-5b and Figures 6a- 6b respectively reveals differences in the detector response. At an applied bias voltage of $-5 \mathrm{~V}$, the cells have a very high charge collection efficiency (approaching $100 \%$ ) in the proton data compared with the same cells in the photon data. This is likely to be an effect from the different ionisation profiles of photon and proton micro beams. The metallisation seems to pick-up a significant fraction of the charge signal at $-40 \mathrm{~V}$, as seen at the left hand side of the strips, due to the high life-time of the charge carriers. The response to proton and photon micro beams is compatible at $-40 \mathrm{~V}$, however the low efficiency in the two rightmost cells in Figure 6d and in the central cell of Figure $5 \mathrm{~b}$ is not understood.

The average collection efficiency for one cell was calculated at each bias voltage for the proton data, as shown in Figure 8 The data features a similar trend as in the photon data, showing an increase in collection efficiency for higher bias voltage, with a plateau at around $(120 \pm 20) \%$ at $\pm 40 \mathrm{~V}$. The high collection efficiency at $\pm 5 \mathrm{~V}$ in the proton data is clearly visible, as well as a slight asymmetry between the positive and negative bias. The average collection efficiency for each of the cells is consistent to within $10 \%$. The calibration uncertainty is estimated at $15 \%$. The measured charge is within the estimated uncertainty consistent with $100 \%$ charge collection efficiency, and no indication of charge multiplication is observed.

Figures $9 \mathrm{a}$ and $9 \mathrm{~b}$ show the response along a line crossing the two read-out strips obtained with photons and protons. Despite the differences in the ionisation profiles, these show similar trends between photon and proton data, such as a reduced efficiency due to the central electrode in the cell in each readout. This is expected since the electrodes are not active areas of the detector. Comparing these two figures shows the charge sharing for each beam data is consistent with the beam width and hence charge sharing is not observed within the limits of the resolution given by the beam width.

\subsection{TRIBIC Results and Simulation}

The time-resolved ion-beam induced current technique (TRIBIC) allows to record transient currents as a function of position on the detector. The current induced by an incident proton was amplified with a Cividec $\mathrm{C} 22 \mathrm{GHz}$ broadband current amplifier [18] and digitised with a WaveMaster-8500 $5 \mathrm{GHz}$ sampling scope from Lecroy. A grid of points was sampled on a single cell shown in Figure 7

The detector set-up was not designed specifically for transient current measurements, resulting in occasional ringing noise in some datapoints. The distortion of the signal was suppressed by applying a Fourier transform to each waveform and isolate the ringing signal with a modal frequency of $6.467 \times 10^{8} \mathrm{~Hz}$ (a period of $1.5 \mathrm{~ns})$.

The experimental results were compared to a numerical simulation to reproduce the principal features found with the TRIBIC measurements. The Synopsys TCAD package was used for the simulations [19].

In a first step a mesh of the detector was created to simulate the electrodes in the diamond 
Study of a 3D diamond detector with photon and proton micro-beams • January $2016 \bullet$ Diamond and Related Materials

bulk, which are modelled with a radius of $2.5 \mu \mathrm{m}$. Only the voltage and the resistance was defined for the electrodes, while the diamond material is assumed to be intrinsically perfect. Boundary conditions are introduced to model the electric potential at the electrodes and in the bulk. The ionising particle is modelled with a non-uniform charge density along its trajectory. The governing equations of semiconductors (Poisson's equation, the electron and hole continuity equations [20]) are then solved in order to simulate how the electrons and holes propagate in the device and the signal induced at the read-out electrode is calculated.

The ionization profile of the $4 \mathrm{MeV}$ protons used in the TRIBIC data was approximated by a density of $5.882 \times 10^{-5} \mathrm{pCum}^{-1}$ from the diamond surface to a depth of $80 \mu \mathrm{m}$, and an higher density of $4.235 \times 10^{-3} \mathrm{pC \mu m}^{-1}$ simulated at a depth between $75-85 \mu \mathrm{m}$ to approximate the Bragg peak. A diamond bandgap energy of $5.5 \mathrm{eV}$ and a dielectric constant of 5.7 were used in the simulation [14]. The simulation input parameters for the modelling of the carrier dynamics were for the electron saturation velocity $v_{s, e}=9.6 \times 10^{6} \mathrm{~cm} / \mathrm{s}$; hole saturation velocity $v_{s, h}=14.1 \times 10^{6} \mathrm{~cm} / \mathrm{s}$; low field electron mobility $\mu_{0, e}=1714 \mathrm{~cm}^{2} \mathrm{~V}^{-1} \mathrm{~s}^{-1}$; and low field hole mobility $\mu_{0, h}=2064 \mathrm{~cm}^{2} \mathrm{~V}^{-1} \mathrm{~s}^{-1}$ [21]. These values are used in a Caughey-Thomas model [22] to calculate the field dependent mobility.

Figures $10 \mathrm{a}$ and $10 \mathrm{~b}$ show a comparison of the TRIBIC experimental data and the simulation partway between a bias and a readout electrode at bias voltages $+60 \mathrm{~V}$ and $-60 \mathrm{~V}$ respectively. The widths of the current pulses are comparable to within $15 \%$, but additional structure is seen in the TRIBIC data. The amount of ringing in the TRIBIC data cannot be completely suppressed, so this structure could possibly be ringing. The simulation curves take into account the average resistance of the graphitic electrodes $(100 \mathrm{k} \Omega)$.

Comparing transient current signals halfway between two electrodes with those near a bias electrode (Figures 11a and 11b indicates that the TRIBIC data follows the same shape predicted by the simulations in the area between bias and readout electrode. Near the electrodes, there is some disagreement between the TRIBIC data and the simulation. This could be due to the remnant ringing in the data (Figure 11a, around $8 \mathrm{~ns}$ ), and imperfections of the electrode formation.

Figures $12 \mathrm{a}$ and $12 \mathrm{~b}$ show the peak amplitudes as a function of position on a quarter cell for the TRIBIC data and the simulation at a bias voltage of $+60 \mathrm{~V}$. A radial symmetry of the peak distribution around the electrodes is observed in both bias polarities corresponding to the shape of the electric field (Figure 12e). Near the readout electrode the highest amplitudes of approximately $50 \mu \mathrm{A}$ are observed, while the lowest peak amplitudes of approximately $15 \mu \mathrm{A}$ are located at the edges of the cell for both bias polarities. The measurements at both bias polarities are compatible within the uncertainty of the measurement. The non-uniform electric field shown in Figure 12e and the non-trivial weighting potential shown in Figure 12f produce the complex shape of the current signal, showing a significant variation with position.

There is weak correspondence in the shape of the distribution of the peak amplitude, but a symmetry along the diagonal is visible for both data and simulation. The strong imbalance in amplitude distribution at the bias electrode and signal electrode visible in data is not reflected in the simulation which shows a more symmetric distribution. This could indicate that the bias electrode had a defect in this particular cell. Qualitatively, a similar shift of the peak amplitude distribution along the diagonal with polarity is observed in both data and simulation. The overall magnitude of peak amplitudes is in the same order of magnitude for data and simulation.

This first comparison is promising for future studies with an optimised set-up and improved reliability of electrodes. Higher quality data obtained can be used to extract information about the charge carrier mobilities and the field distribution within the detector.

\section{Conclusions}

A 3D diamond detector was produced with varying laser processing parameters. The detector was characterised electronic and structural properties were investigated, and the response 
Study of a 3D diamond detector with photon and proton micro-beams • January $2016 \bullet$ Diamond and Related Materials

to a photon and proton micro-beam was studied. The laser processing parameters affect the electrode diameter and stress induced in the bulk diamond, but only a minor effect on the resistivity has been observed. Raman studies indicate that the graphitic content in the electrodes is high. The results with particle beams are consistent with full charge collection within the active regions of the detector. No significant charge sharing between neighbouring cells has been observed. Transient current measurements have been performed spatially resolved on a single $3 \mathrm{D}$ diamond detector cell and have been compared to simulations. Qualitatively fair agreement is observed, but further improvement in the electrode fabrication and optimisation of the TRIBIC set-up are needed to extract precision data on carrier dynamics and electric field distribution.

\section{ACKNowledgements}

The authors would like to thank all members at Diamond Light Source for their help in acquiring the photon data. $\mathrm{AO}$ would like to acknowledge support from the Royal Society through grant UF120106.

\section{REFERENCES}

[1] A. Oh, B. Caylar, M. Pomorski, and T. Wengler, "A novel detector with graphitic electrodes in CVD diamond," Diamond and Related Materials, 38, 2013.

[2] S. Lagomarsino, M. Bellini, C. Corsi, F. Gorelli, G. Parrini, M. Santoro, and S. Sciortino, "Three-dimensional diamond detectors: Charge collection efficiency of graphitic electrodes," Applied Physics Letters 103, 2013.

[3] F. Bachmair, L. Bä, P. Bergonzo, B. Caylar, G. Forcolin, I. Haughton, D. Hits, H. Kagan, R. Kass, L. Li, A. Oh, S. Phan, M. Pomorski, D. S. Smith, V. Tyzhnevyi, R. Wallny, and D. Whitehead, "A 3D diamond detector for particle tracking," NIM Phys A 786, 2015.
[4] B. Caylar, M. Tomasz, and P. Bergonzo, "Laser-processed three dimensional graphitic electrodes for diamond radiation detectors," Applied Physics Letters 103, 2013.

[5] S. Lagomarsino, M. Bellini, C. Corsi, V. Cindro, K. Kanxheri, A. Morozzi, D. Passeri, L. Servoli, C. J. Schmidt, and S. Sciortino, "Radiation hardness of threedimensional polycrystalline diamond detectors," Applied Physics Letters 106, 2015.

[6] T. V. Kononenko, M. Meier, M. S. Komlenok, S. M. Pimenov, V. Romano, V. P. Pashinin, and V. I. Konov, "Microstructuring of diamond bulk by IR femtosecond laser pulses," Applied Physics A 90, 2008.

[7] T. V. Kononenko, M. S. Komlenok, V. P. Pashinin, S. M. Pimenov, V. I. Konov, M. Neff, V. Romano, and W. Lüthy, "Femtosecond laser microstructuring in the bulk of diamond," Diamond and Related Materials 18, 2009.

[8] Coherent Inc., "Libra Series Data Sheet." http://www.coherent.com/downloads/ LibraSeries_CoherentDataSheet_revC_ May2013_4.pdf, 2014.

[9] Element Six Ltd., "Properties of Electronic Grade CVD Diamonds," 2014.

[10] Textronix, "Keithley Sourcemeter Data Sheet." http://www.keithley. com/products/software/?path=2410/ Documents\#4, 2015. [Accessed 01-Nov2015].

[11] HORIBA Ltd., "LabRAM HR Evolution Raman Spectrometer." http: //www.horiba.com/uk/scientific/ products/raman-spectroscopy/ raman-spectrometers/ raman-microscopes/hr-evolution/ labram-hr-evolution-17309/ 2015. [Accessed 24-June-2015].

[12] S. Reich and C. Thomsen, "Raman spectroscopy of graphite," Royal Society Publishing 362, 2004. 
Study of a 3D diamond detector with photon and proton micro-beams • January $2016 \bullet$ Diamond and Related Materials

[13] K. J. S. Sawhney, I. P. Dolbnya, M. K. Tiwari, L. Alianelli, S. M. Scott, G. M. Preece, U. K. Pederson, and R. D. Walton, "A test beamline on diamond light source," in AIP Conf Proc, 1234 (A. Publishing, ed.), 2010.

[14] L. S. Pan, S. Han, and D. R. Kania, Diamond: Electronic Properties and Applications. Kluwer Academic, Dordrecht, 1995.

[15] J. H. Hubbell and S. M. Seltzer, "XRay Mass Attenuation Coefficients for Elemental Media." http://www.nist.gov/ pml/data/xraycoef/, 2004. [Accessed 28April-2015].

[16] M. Jakšić, I. Bogdanović Radović, M. Bogovac, V. Desnica, S. Fazinić, M. Karlušić, Z. Medunić, H. Muto, Z. Pastuović, Z. Siketić, N. Skukan, and T. Tadić, "New capabilities of the Zagreb ion microbeam system," NIM Phys B, 260, 2007.

[17] J. Ziegler, "Stopping and Range of Ions in Matter." http://www.srim.org/, 2013. [Accessed 23-Nov-2015].
[18] CIVIDEC Instrumentation, "Current Amplifier Data Sheet." https://cividec.at/ files/15_pdf.pdf 2015. [Accessed 01Nov-2015].

[19] Synopsys Inc., "Synopsys TCAD." http: //www.synopsys.com/home.aspx/, 2015. [Accessed 28-April-2015].

[20] S. M. Sze, Semiconductor Devices Physics and Technology. John Wiley and Sons, 1985.

[21] H. Pernegger, S. Roe, P. Weilhammer, V. Eremin, H. Frais Kölbl, E. Griesmayer, H. Kagan, S. Schnetzer, R. Stone, W. Trischuk, D. Twitchen, and A. Whitehead, "Charge-carrier properties in synthetic single-crystal diamond measured with the transient-current technique," $A p$ plied Physics 97, 2005.

[22] D. M. Caughey and R. E. Thomas, "Carrier mobilities in silicon empirically related to doping and field," in Proc. IEEE vol 52 (IEEE, ed.), 1967. 
Study of a 3D diamond detector with photon and proton micro-beams • January $2016 \bullet$ Diamond and Related Materials

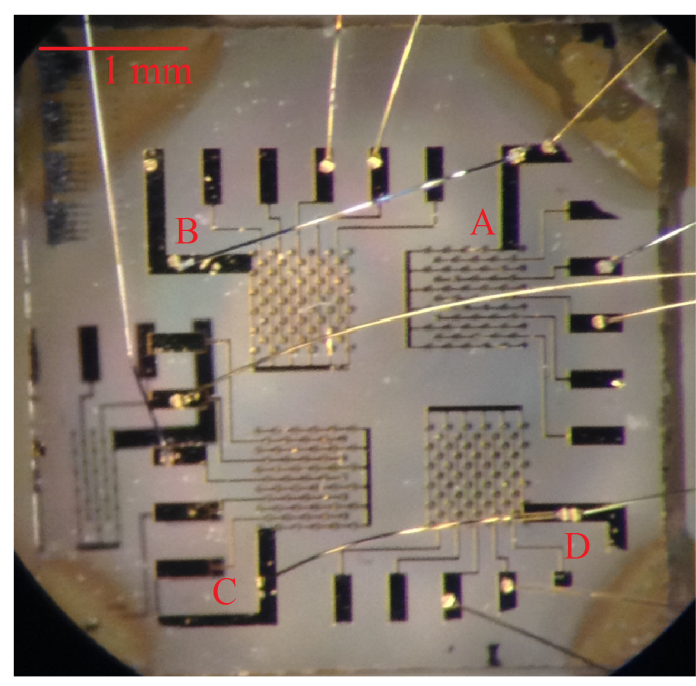

(a)

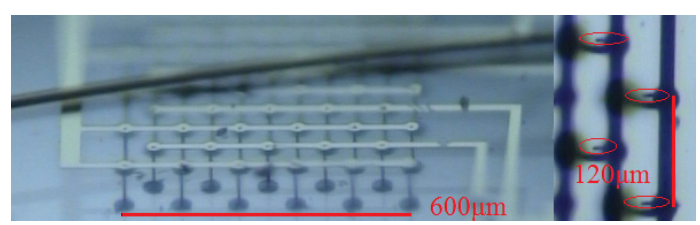

(c)

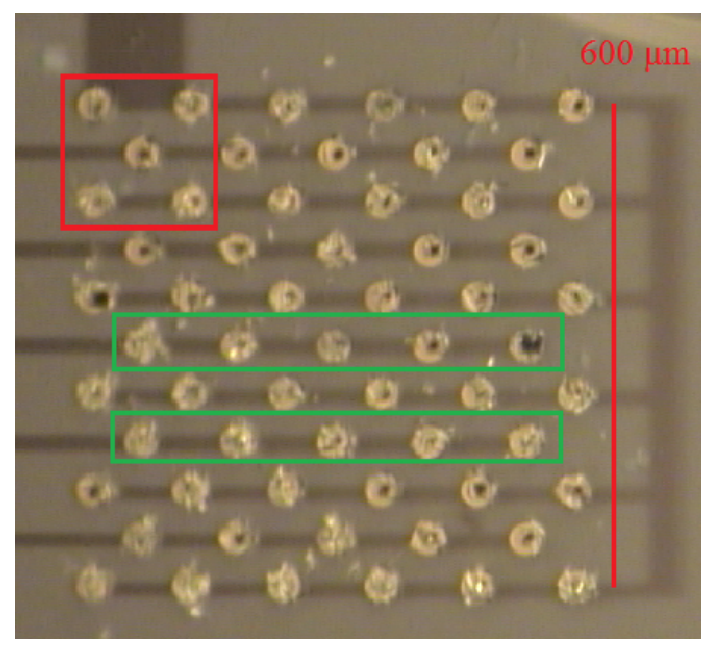

(b)

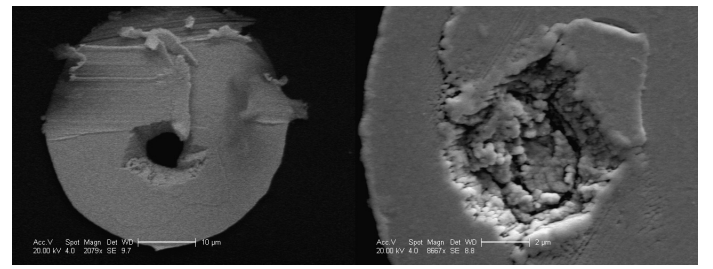

(d)

Figure 1: (a) Optical image of the detector. The four arrays are referred to as (from top right anti-clockwise) $A, B, C$, and $D$. (b) Array $A$ on the detector. The readout of two neighboring strips as described in the text are outlined in green, and a basic cell is outlined in red. (c) Electrodes fabricated on a detector within array $A$ (left) and the presence of secondary electrodes observed in array $C$ after processing the diamond, highlighted in red (right). (d) Scanning Electron Microscope (SEM) images of one electrode in array B, showing the exit side (left) and the seed side (right) of the electrode. 
Study of a 3D diamond detector with photon and proton micro-beams • January $2016 \bullet$ Diamond and Related Materials

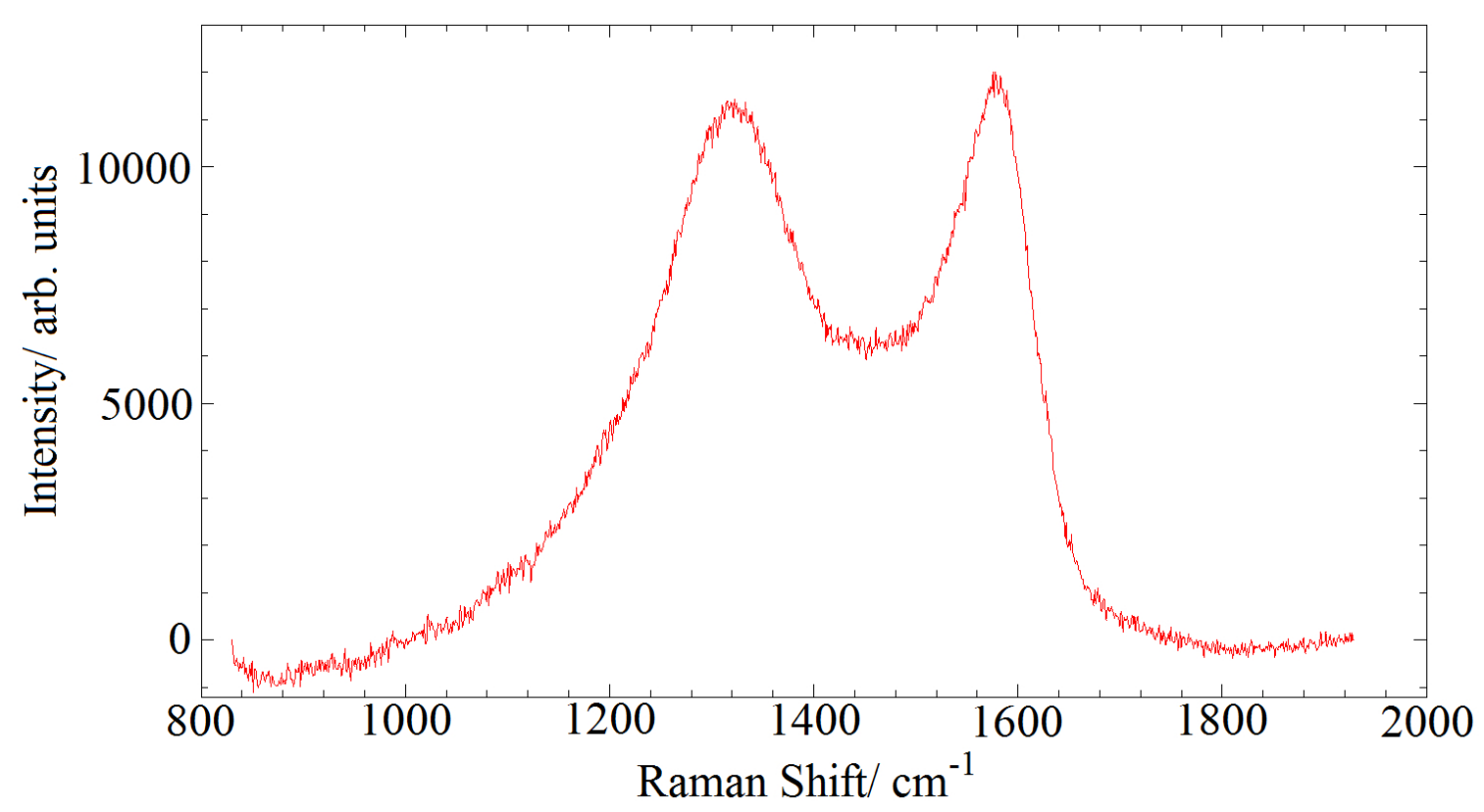

Figure 2: Raman spectroscopy of one electrode, with the baseline subtracted. The grating dispersion is $1.3 \mathrm{~cm}^{-1}$, with a pixel resolution of $5.17 \mathrm{~cm}^{-1}$. 
Study of a 3D diamond detector with photon and proton micro-beams • January $2016 \bullet$ Diamond and Related Materials

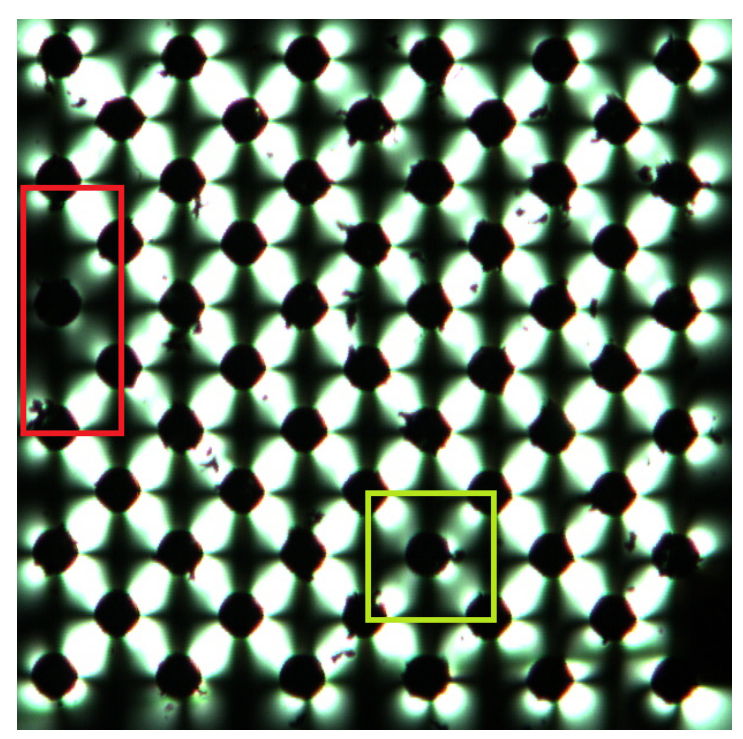

(a)

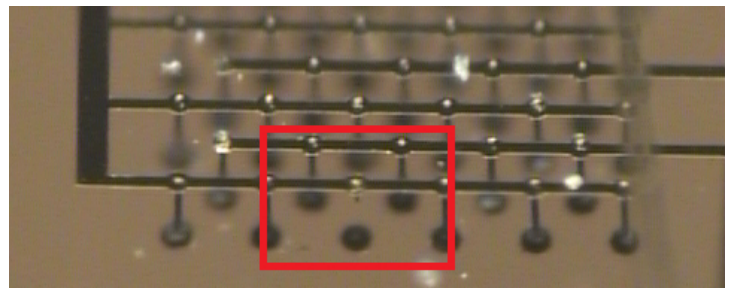

(b)

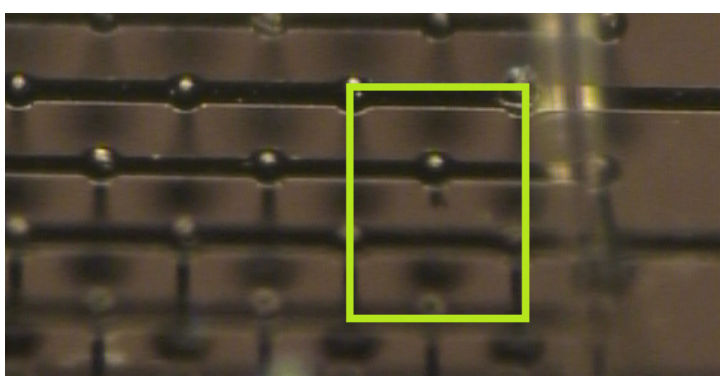

(c)

Figure 3: (a) Cross-polarised image of array B. High brightness areas show high levels of stress within the array. Partially formed electrodes are highlighted in red and green. (b) and (c) Malformed electrodes identified from Figure $3 a$. 
Study of a 3D diamond detector with photon and proton micro-beams • January $2016 \bullet$ Diamond and Related Materials

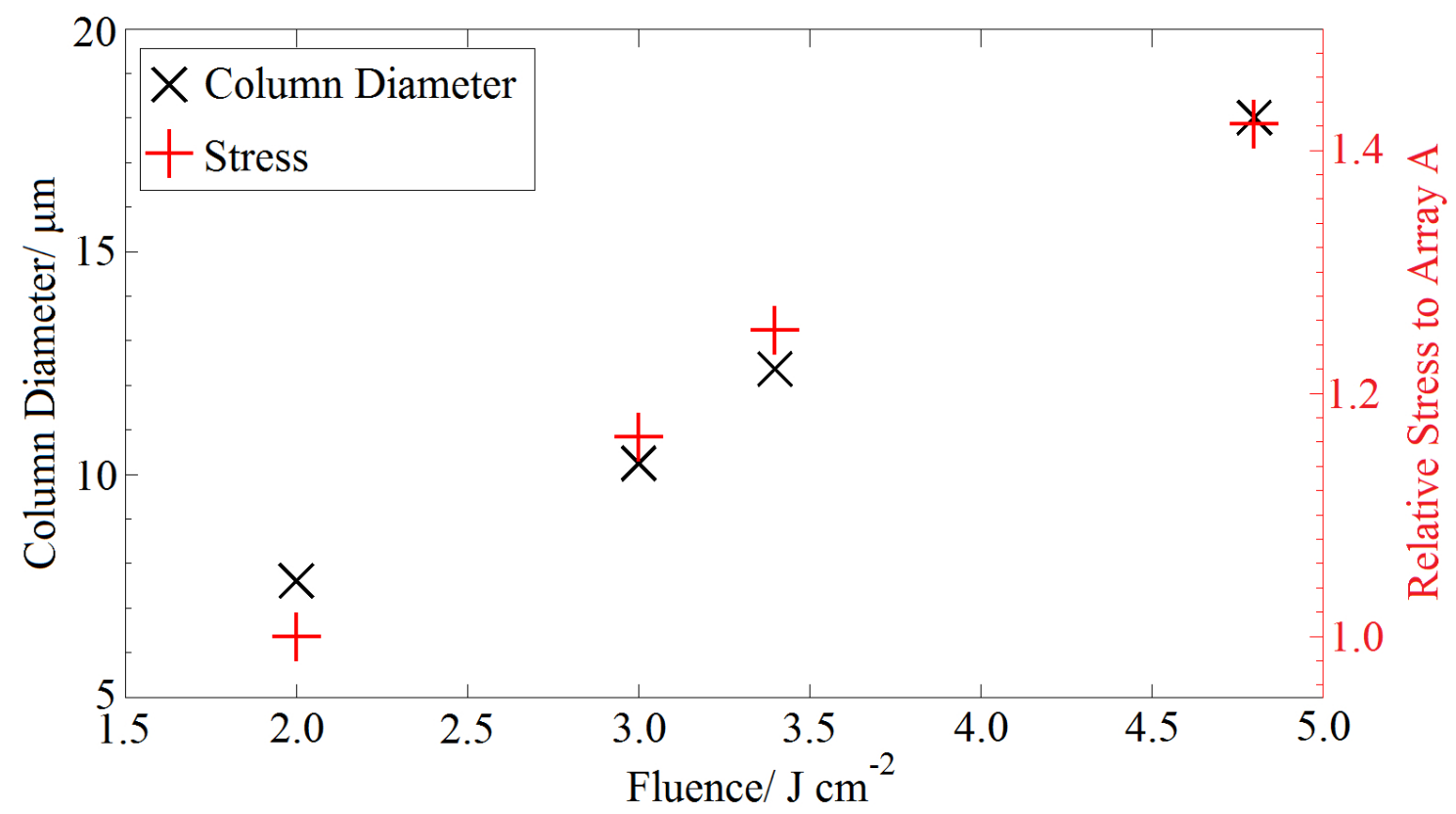

Figure 4: The beam fluence used to fabricate each array versus average electrode diameter (black) and stress (red).

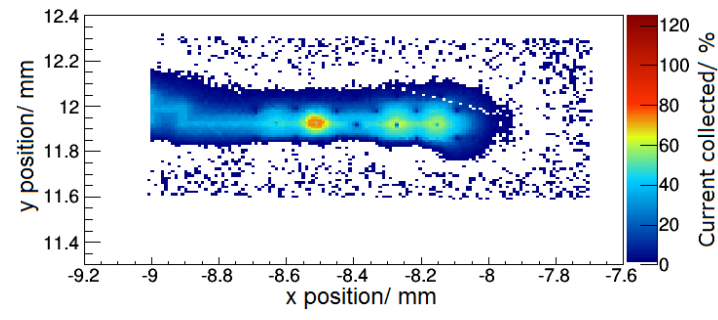

(a)

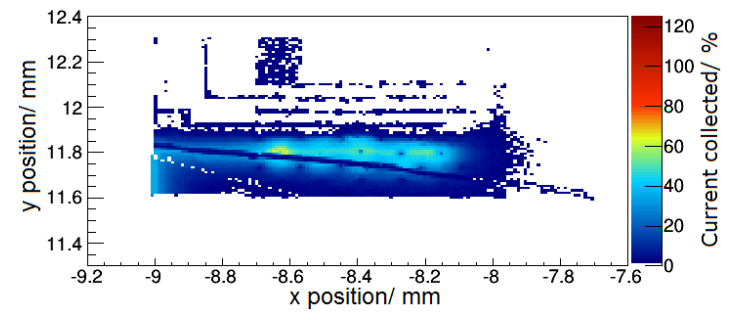

(c)

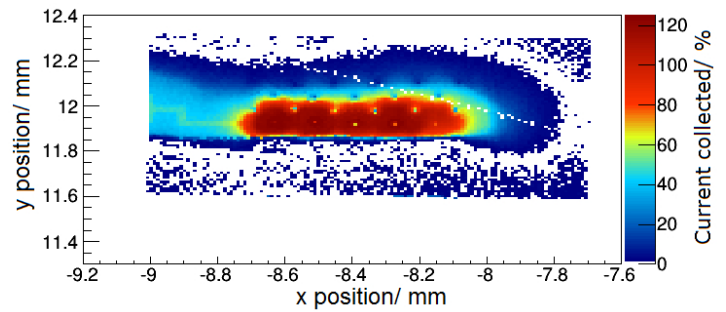

(b)

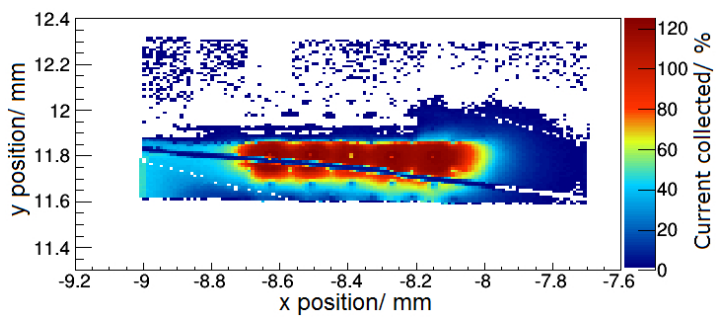

(d)

Figure 5: Detector response to a $15 \mathrm{keV}$ photon beam for array A for two different readouts channels at two different bias voltages. Readout channel one at (a) $-5 \mathrm{~V}$ and (b) $-40 \mathrm{~V}$. Readout channel two at (c) $-5 \mathrm{~V}$ and $(d)-40 \mathrm{~V}$. 
Study of a 3D diamond detector with photon and proton micro-beams • January $2016 \bullet$ Diamond and Related Materials

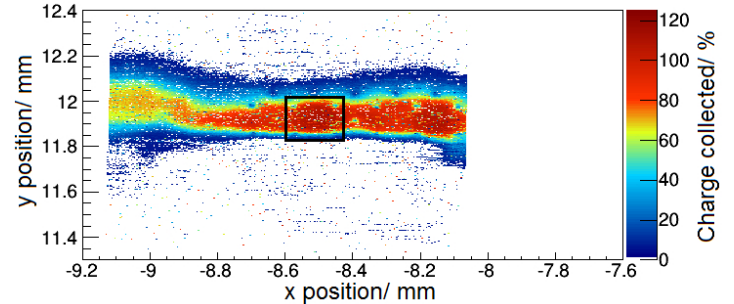

(a)

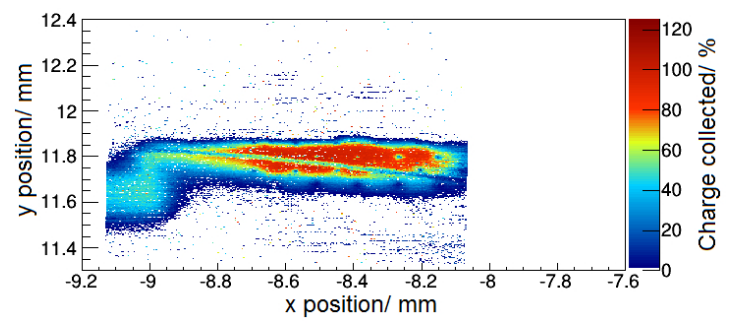

(c)

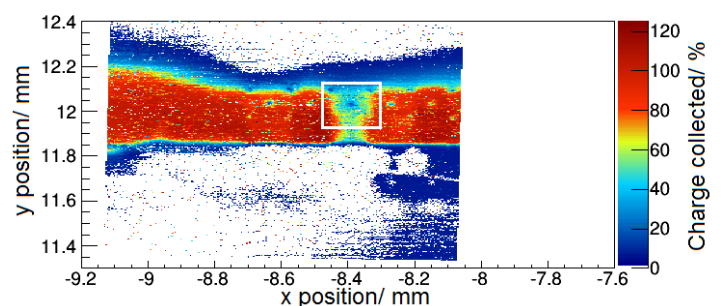

(b)

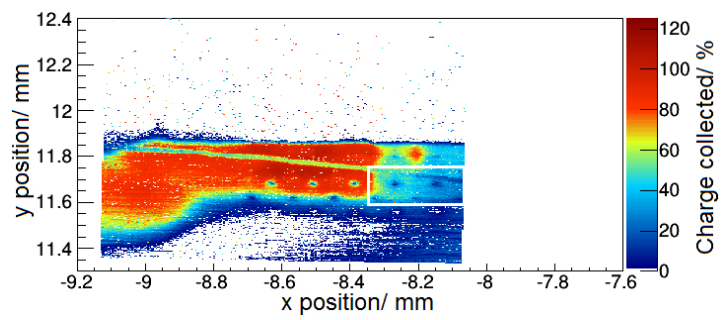

(d)

Figure 6: Signal response to proton micro-beam of array A with two readout channels at two bias voltages. Readout one at (a) $-5 \mathrm{~V}$ and (b) $-40 \mathrm{~V}$. Readout two at (c) $-5 \mathrm{~V}$ and (d) $-40 \mathrm{~V}$. The area highlighted in black in (a) is the cell shown in Figure 7 , and the areas highlighted in white in (b) and (d) indicate cells with a low collection efficiency.

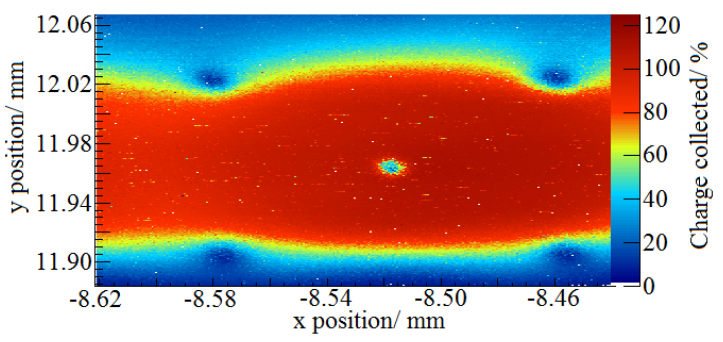

Figure 7: Signal response to proton micro-beam for a single cell on array $A$ with a bias voltage of $-5 \mathrm{~V}$.

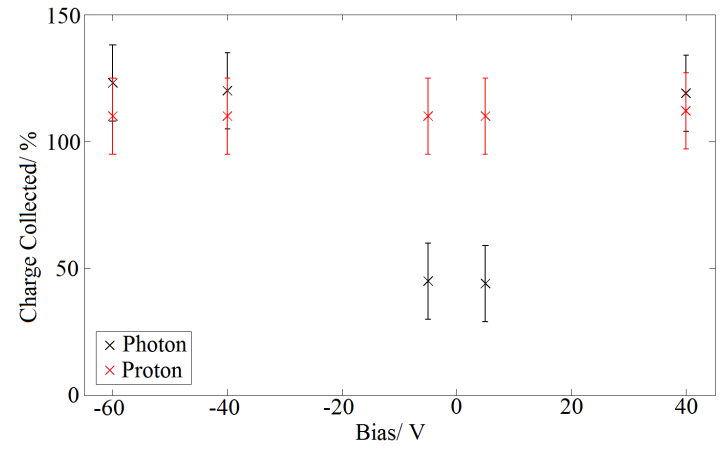

Figure 8: Average collection efficiency as a function of bias voltage for the highlighted cell in Figure 6a, for photon (black) and proton (red) data. 
Study of a 3D diamond detector with photon and proton micro-beams • January $2016 \bullet$ Diamond and Related Materials

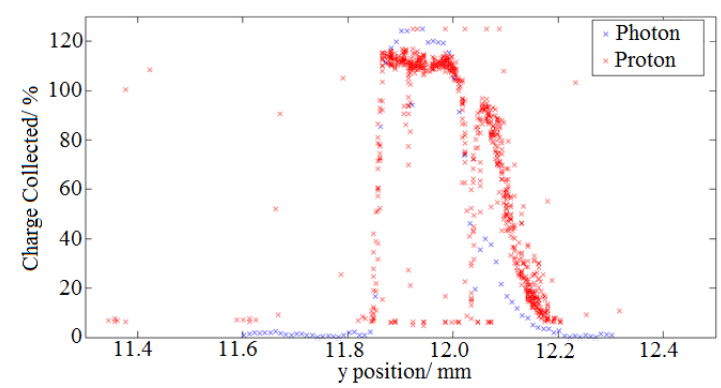

(a)

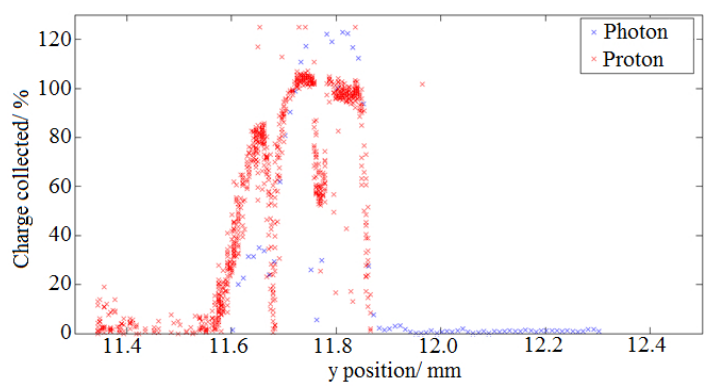

(b)

Figure 9: Signal response to photon and proton micro-beams along a line at $x=-8.51 \mathrm{~mm}$ at a bias voltage of $-40 \mathrm{~V}$ for (a) readout one, and (b) readout two.

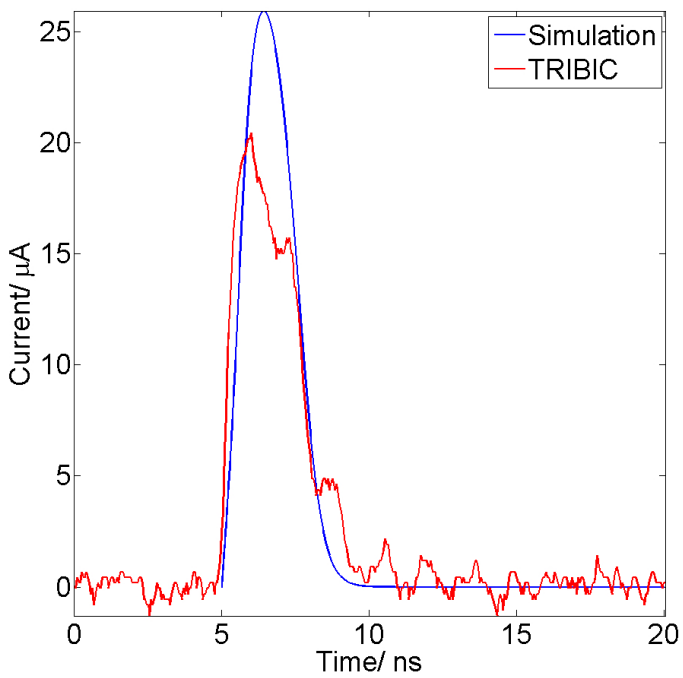

(a)

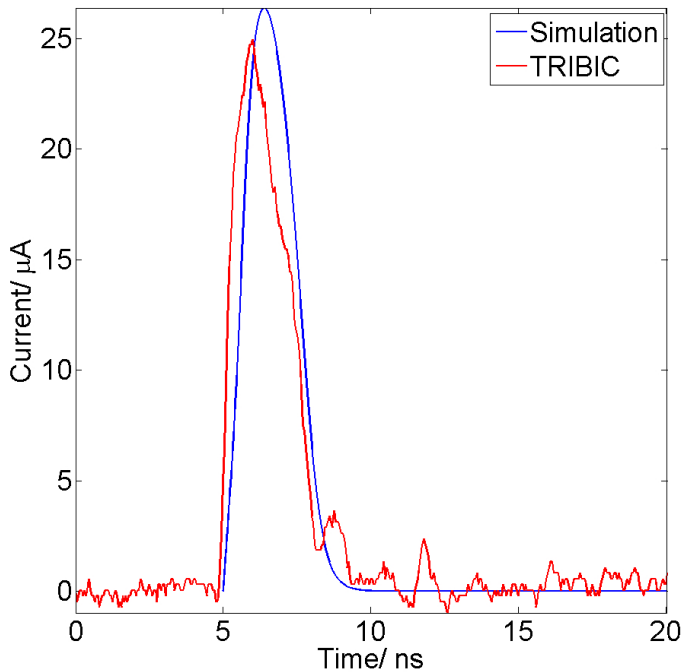

(b)

Figure 10: Transient current signal from TRIBIC data and simulation at the center of the diagonal between the bias and readout electrode for a bias voltage of (a) $+60 \mathrm{~V}$ and (b) $-60 \mathrm{~V}$. 
Study of a 3D diamond detector with photon and proton micro-beams • January $2016 \bullet$ Diamond and Related Materials

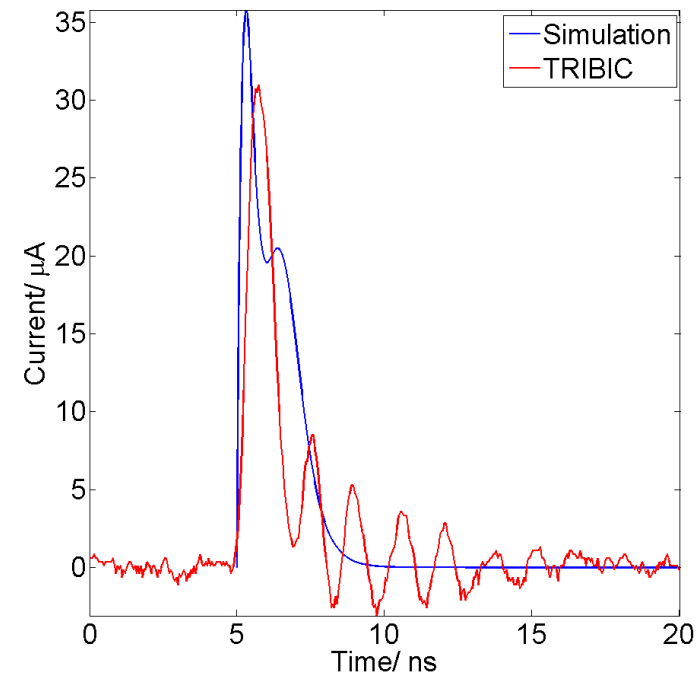

(a)

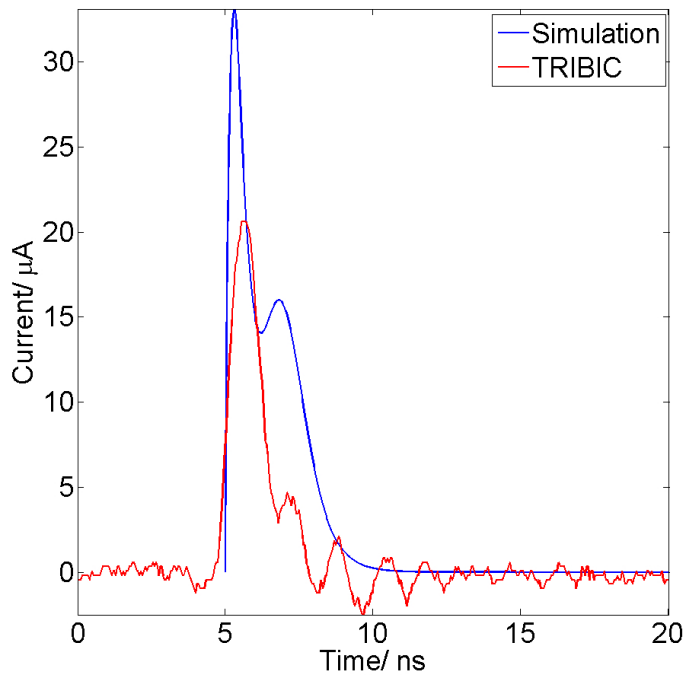

(b)

Figure 11: Transient current signal from TRIBIC data and simulation at a bias electrode for a bias voltage of (a) $+60 \mathrm{~V}$ and $(b)-60 \mathrm{~V}$. 
Study of a 3D diamond detector with photon and proton micro-beams • January $2016 \bullet$ Diamond and Related Materials

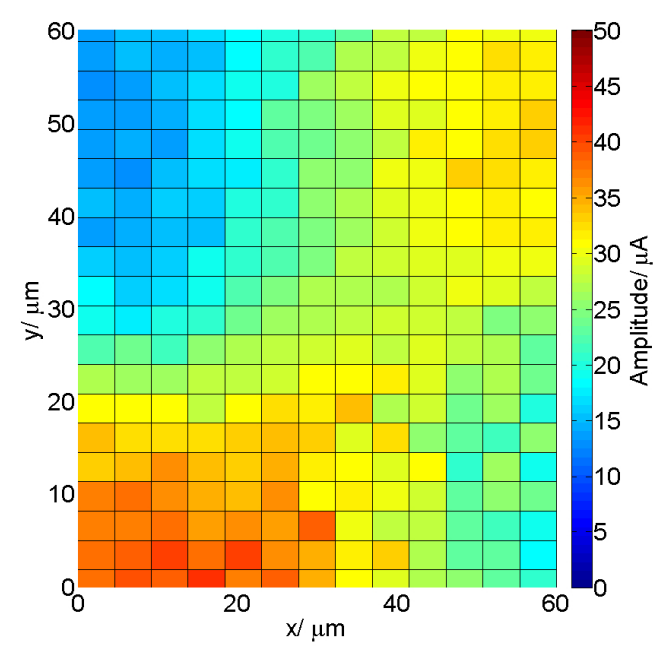

(a)

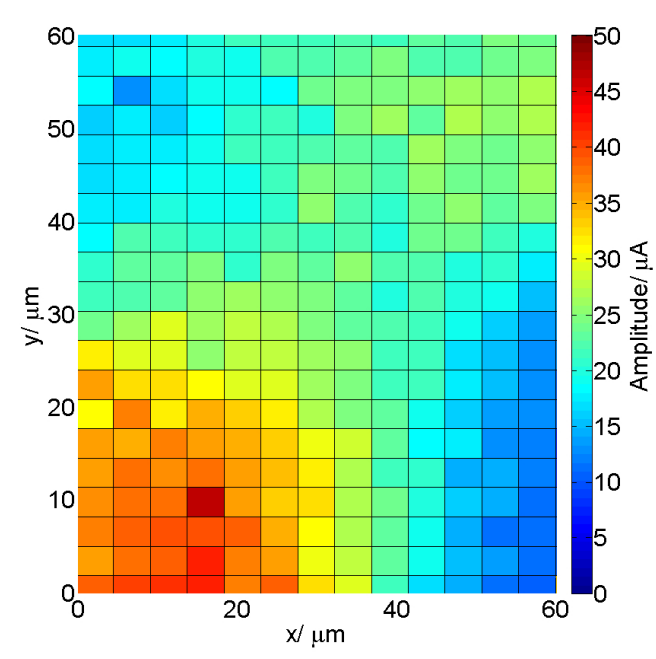

(c)

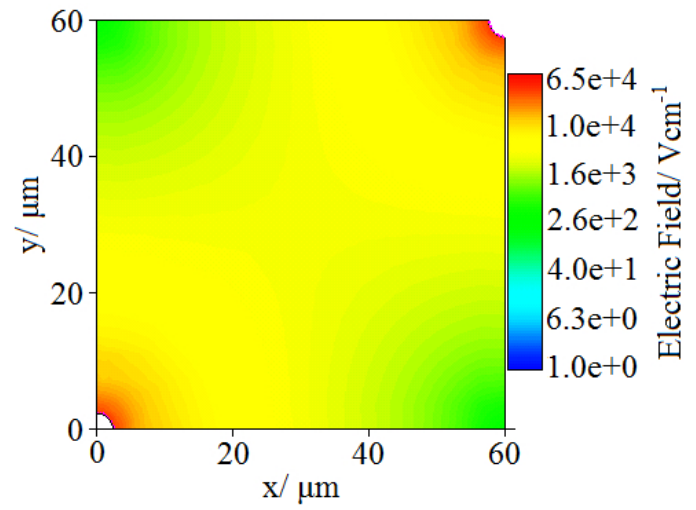

(e)

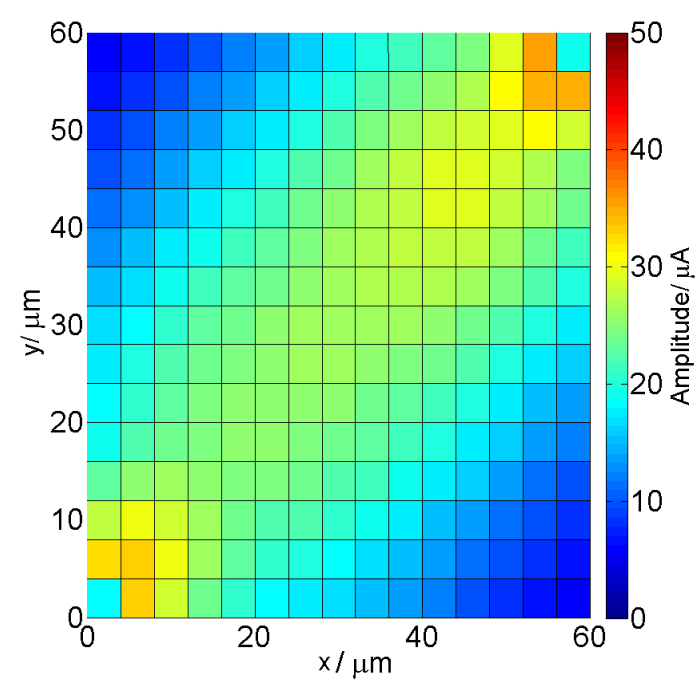

(b)

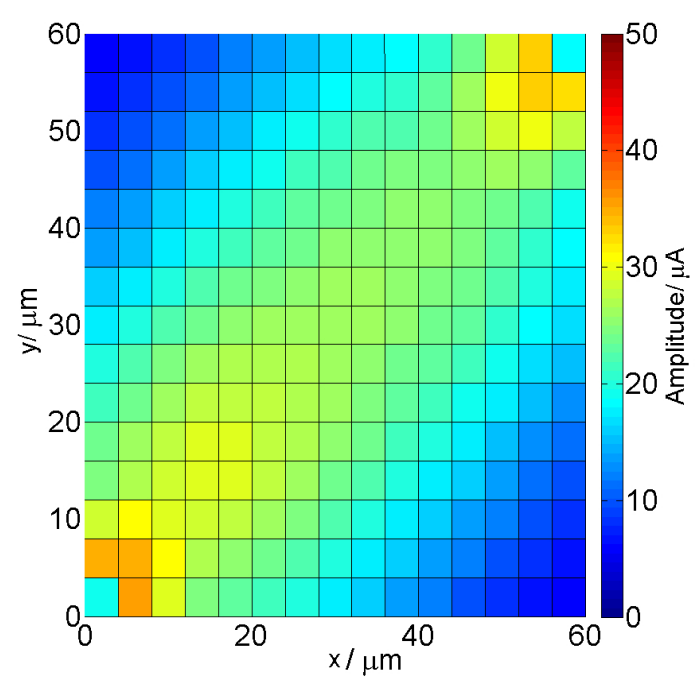

(d)

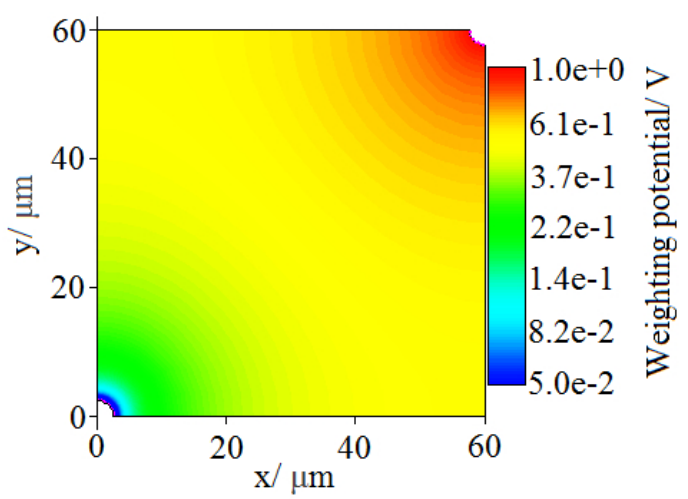

(f)

Figure 12: Peak amplitude as a function of position for the top right quarter cell for TRIBIC data (a,c) and simulation $(b, d)$ for a bias voltage of $+60 \mathrm{~V}(a, b)$ and $-60 \mathrm{~V}(c, d)$. For the simulations the electrix field distribution (e) and the weighting potential ( $f)$ were used at a depth of $250 \mu \mathrm{m}$ The bias and readout electrodes are located at $(60,60)$ and $(0,0)$, respectively. 